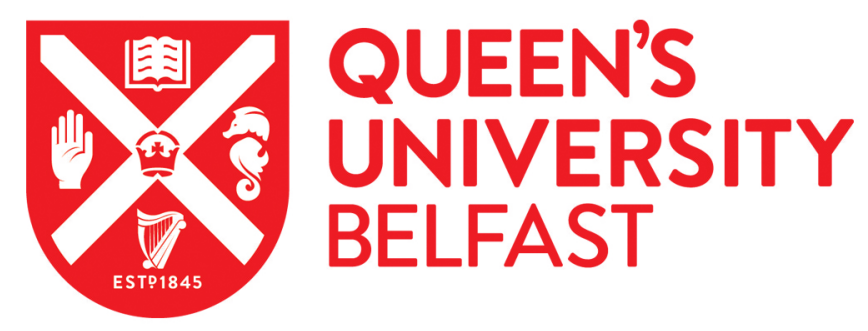

\title{
A Randomized Combined Channel Approach for the Quantification of Color- and Intensity-Based Assays with Smartphones
}

Neils, J., Zhao, YJ., Bura, L., Rafferty, K., Elliott, C., \& Campbell, K. (2020). A Randomized Combined Channel Approach for the Quantification of Color- and Intensity-Based Assays with Smartphones. Analytical Chemistry, 92(11), 7852. https://doi.org/10.1021/acs.analchem.0c01099

Published in:

Analytical Chemistry

Document Version:

Peer reviewed version

Queen's University Belfast - Research Portal:

Link to publication record in Queen's University Belfast Research Portal

Publisher rights

(C) 2020 American Chemical Society. This work is made available online in accordance with the publisher's policies. Please refer to any applicable terms of use of the publisher.

\section{General rights}

Copyright for the publications made accessible via the Queen's University Belfast Research Portal is retained by the author(s) and / or other copyright owners and it is a condition of accessing these publications that users recognise and abide by the legal requirements associated with these rights.

Take down policy

The Research Portal is Queen's institutional repository that provides access to Queen's research output. Every effort has been made to ensure that content in the Research Portal does not infringe any person's rights, or applicable UK laws. If you discover content in the Research Portal that you believe breaches copyright or violates any law, please contact openaccess@qub.ac.uk. 


\section{Article}

\section{A randomised combined channel approach for the quantification of colour and intensity based assays with smartphones}

Joost Laurus Dinant Nelis, Yunfeng Zhao, Laszlo Bura, Karen Rafferty, Christopher T. Elliott, and Katrina Campbell

Anal. Chem., Just Accepted Manuscript • DOI: 10.1021/acs.analchem.0c01099 • Publication Date (Web): 08 May 2020

Downloaded from pubs.acs.org on May 12, 2020

\section{Just Accepted}

"Just Accepted" manuscripts have been peer-reviewed and accepted for publication. They are posted online prior to technical editing, formatting for publication and author proofing. The American Chemical Society provides "Just Accepted" as a service to the research community to expedite the dissemination of scientific material as soon as possible after acceptance. "Just Accepted" manuscripts appear in full in PDF format accompanied by an HTML abstract. "Just Accepted" manuscripts have been fully peer reviewed, but should not be considered the official version of record. They are citable by the Digital Object Identifier (DOI®). "Just Accepted" is an optional service offered to authors. Therefore, the "Just Accepted" Web site may not include all articles that will be published in the journal. After a manuscript is technically edited and formatted, it will be removed from the "Just Accepted" Web site and published as an ASAP article. Note that technical editing may introduce minor changes to the manuscript text and/or graphics which could affect content, and all legal disclaimers and ethical guidelines that apply to the journal pertain. ACS cannot be held responsible for errors or consequences arising from the use of information contained in these "Just Accepted" manuscripts. 


\title{
A randomised combined channel approach for the quantification of colour and intensity based assays with smartphones
}

\author{
Joost L.D. Nelis ${ }^{+*}$, Yunfeng Zhao ${ }^{+}$, , Laszlo Bura ${ }^{\dagger}$, Karen Rafferty ${ }^{\ddagger}$, Christopher T. Elliott ${ }^{+}$and Katrina \\ Campbell ${ }^{+}$ \\ +· Institute for Global Food Security, School of Biological Sciences, Queen's University of Belfast, 19 Chlorine Gardens, \\ Belfast, BT9 5DL, UK. $\uparrow$. Department of Food and Drug, University of Parma, Parco Area delle Scienze 27/A 43124, Parma, \\ Italy. $\$$ School of Electronics, Electrical Engineering and Computer Science, Queen’s University Belfast, 125 Stranmillis \\ Road, Belfast, BT9 5AH, UK. *Corresponding author, email: J.Nelis@qub.ac.uk ; phone: +442890976543.
}

\begin{abstract}
Quantification of colorimetric assays with smartphones is being increasingly reported. However, a complete characterisation of the performance of existing colour spaces and single colour channels for optimum colour/intensity change quantification is absent. Moreover, it has not been ascertained if it is necessary to utilize existing colour spaces to reach optimal assay quantification. In this study a randomised channel approach was adapted utilizing all single channels from RGB, HSV and CieLab colour space and all non-repeating random combinations of 2 and 3 channels of these colour spaces. Assays based on colour or intensity change using $\mathrm{pH}$ strips and gold or carbon black nanoparticle containing paper strips were optimized using this approach. Several novel channel combinations showed great promise in terms of prediction error and inter-phone variation reduction, outperforming RGB, HSV and CieLab colour spaces. These novel combinations were used in a custom developed smartphone application that performed automated background subtraction and polynomial regression for the quantification of a lateral flow assay for the detection of goat milk adulteration with cow milk and for $\mathrm{pH}$ prediction in soil. For the lateral flow assay the channel combination BSA was found optimum (mean average error $36 \pm 6 \%$; linear regression $\mathrm{R}^{2}=0.97$ ). For the soil $\mathrm{pH}$ assay the channel combination RLC was found optimum (mean average error $1.31 \pm 0.02 \%$; linear regression $\mathrm{R}^{2}=0.997$ ). The study has shown that non-classical channel combinations for colorimetric quantification of specific assays are very promising and should be considered for smartphone-based analysis.
\end{abstract}

The fourth industrial revolution is heralding a paradigm shift in diagnostics by combining state-of-the-art biotechnology and lab-on-a-chip engineering with cloud computing. Smartphones are especially attractive for this application since they combine the necessary features for portable sensors (e.g. processing power, and connectivity) with simple interfacing through use of an app. Moreover, smartphones are ubiquitous and equipped with GPS, which facilitates target mapping in remote locations ${ }^{1-3}$. Smartphone-based sensors have been developed for a range of applications including disease diagnostics, patient wellbeing, environmental monitoring and food contaminant/allergen screening ${ }^{4-8}$. The large majority of these systems utilize the camera for colorimetric sensing. Photometric data needs to be extracted from images to correlate a change in assay colour change or intensity change to target concentration.

The colour system used in the CMOS image sensors of smartphones is the red green blue (RGB) system. This colour system can be converted to other colour systems, the most common of which are: hue saturation value (HSV) and CieLAB colour spaces. Colour/intensity change can be correlated to one, two or three of the channels in these colour spaces. However, colour/intensity changes are split between these channels differently. In the RGB model a colour is defined by the position in a cube with $\mathrm{x}, \mathrm{z}$, and $\mathrm{y}$ coordinates indicating the red, green and blueness of the colour. In the HSV model, hue represents pure colours and changes over an angle from 0 to 360 degrees around a cone. Saturation ranges from $0 \%$ to $100 \%$ (horizontal cone axe) and indicates the range of grey in the colour. Value (also called brightness with the abbreviation HSB instead of HSV) runs from $0 \%$ to $100 \%$ along the vertical axe of the cone and represents colour brightness. CieLAB (called LAC from here on to avoid confusion with the $\mathrm{B}$ channel in RGB) is based on a spherical model with the vertical axe (L) representing lightness (from black $(0 \%)$ to white $(100 \%))$. A and $\mathrm{C}$ axes (perpendicular axes in the orthodrome of the sphere) generally run from -128 to 127 and represent green to red and blue to yellow colours respectively. These axes represent the existing opposing colours that cannot be combined. Defining colour as a combination of these axes allows digitalising all real colours perceived by the human eye. Further information about colour spaces can be found in ${ }^{9-11}$.

Conversion to a certain colour space/channel for a specific assay seems logical since different colour spaces divide colours differently. Thus, picking the right channel can potentially filter out noise for a given assay. For instance, if the assay is based on colour change then hue seems an appropriate choice. For a colour intensity change however, lightness of LAC or value or saturation of HSV seem a better choice. Indeed several of the citation classics (defined here as $>100$ citations) treating smartphone-based colorimetrics utilize hue to measure colour change ${ }^{12-14}$. Other studies used $\mathrm{V}$ or $\mathrm{S}$ to measure intensity ${ }^{15,16}$. Another classic reported the combination of chromaticity channels $\mathrm{A}$ and $\mathrm{C}$ for colour change quantification ${ }^{17}$. Although these works have pioneered smartphone colorimetrics several 
interesting questions remain to be investigated. One such question is whether combining the three channels in each colour space would be beneficial for the correlation of colour/intensity change to target concentration in a given assay. On one hand error may be introduced to the measurement if a channel is integrated that does not reflect the process measured in the assay. On the other hand, valuable information may be lost. Moreover, the question could be raised if it is possible to detect both colour and colour intensity change with the same system. Lastly, colour spaces have been optimized to define the expression of real colours. However, they have not been optimized to quantify a specific colour/intensity change in a specific assay. It is thus possible that other optimum channel combinations exist as the combinations possible within each colour space for the quantification of a specific assay.

The literature suggests that combining 3 channels of a colour space can deliver good performance ${ }^{18-22}$. Overall these studies showed that LAC outperforms RGB for colour change measurements 19,22. However, no such comparisons were identified for colour intensity change even though most colorimetric assays (such as lateral flow assays (LFA)) are based on that principle. Additionally, no studies were identified comparing performance of the individual channels of $\mathrm{HSV}$, RGB and LAC and the respective colour spaces combining these channels for intensity or colour change based assays. However, the performance of individual channels for colour quantification clearly differs in RGB, HSVand LAC colour space with various channels contributing little to the analytical signal in several assays ${ }^{23-27}$. Thus, it is unclear if combining channels is beneficial for colour/intensity change quantification. Moreover, to our knowledge, it has not been investigated if it is possible to use a specific channel combination for the quantification of colour and intensity changes simultaneously, nor has there been a study investigating if alternative channel combinations using combinations of two or three channels from various colour spaces can outcompete the use of classical colour spaces for the quantification of specific assays.

Another interesting point is the effect of variation in background and source (smartphone flashlight) illumination on smartphone-based colour quantification. The spectral profile of various flashlights may differ. This can affect the absorption/transmission profile of a colorimetric assay. Regarding background illumination, a light-shielding box is sometimes used to reduce variation in background illumination 28,29,6,30. However others have shown that a box can be avoided through algorithmic corrections ${ }^{31}$. Avoiding use of a lightshielding box would be beneficial in terms of maintaining easeof-use and in-the-pocket portability for the end-user. Another point is unequal sample illumination for which the use of optical diffuser to obtain equal illumination was argued (although not tested) to be beneficial ${ }^{14}$. Moreover, Kim et al., showed that optical diffuser effectively eliminated unequal sample illumination $^{32}$. Equally, background illumination correction may avoid the need for such diffusers.

In the present study a comprehensive characterization of the effects that colour channel choices and illumination have on smartphone-based colour analyses was conducted. These analyses allowed clear conclusions to be reached regarding optimum channel choice for smartphone-based image analyses as well as shed light on the effects of source/background illumination on the measurement error. An application was developed for colour/intensity change quantification based on the use of a pixel wise background illumination algorithm and a prediction algorithm using polynomial regression. Usefulness of a light-shielding box and optical diffuser for colour/intensity predictions with this application was tested. The performance of individual channels of RGB, HSV and LAC colour space for colour ( $\mathrm{pH}$ strips) and intensity (filter papers with various nanoparticles and LFA) quantification was compared with random novel combinations of any 2 or 3 of these channels and classical LAC, HSV and RGB colour space. Performance of the identified optimum spaces was compared between various phone models and robustness towards background and source illumination variation was investigated. The optimized systems were then used for $\mathrm{pH}$ determination in soil samples and bovine milk quantification in goat milk.

\section{MATERIALS AND METHODS}

\section{Materials}

A full description of the materials used is given in the supplementary information. The mobile phone models used were: a Huawei P8 Lite (Huawei), Samsung Galaxy J7 (Samsung), iPhone 7 (iPhone) and Samsung Galaxy Tab E (Tablet).

\section{Statistical analyses}

Data analyses was performed with GraphPad Prism 6 Software. Barlett's and Brown-Forsythe tests were used to test if standard deviation (STD) differed between columns in a oneway ANOVA. For post-hoc analyses, a one-way ANOVA Tukey's multiple comparison test was used. For two-way ANOVA Sidak's multiple comparison test was used. $p$-values were corrected for multiplicity. Mean average error (MAE) was calculated on the totality of predictions over the concentration ranges mentioned above.

\section{Nanoparticle synthesis}

Piranha solution and aqua regia were used to remove residues from glassware. Gold nanoparticles (GNP) were synthesized following ${ }^{33}$. Briefly, $500 \mu \mathrm{L} \mathrm{HAuCl}_{4}(100 \mathrm{mM})$ and $194.5 \mathrm{ml}$ $\mathrm{H}_{2} \mathrm{O}$ was brought to boil in a round bottom flask under stirring. Sodium citrate solution $(5 \mathrm{ml} ; 1 \%(\mathrm{w} / \mathrm{v}))$, was added at boiling point and the mixture was left boiling for 30 minutes while stirring then left to cool down. GNP concentration $(2.8 \mathrm{nM})$ was estimated following ${ }^{34}$. UV-VIS measurements and GNP size estimations used for the estimation are reported elsewhere ${ }^{35}$.

\section{pH strips}

$0.1 \mathrm{M}$ buffers systems: Citrate-phosphate ( $\mathrm{pH}$ 5.0, 5.5), phosphate $(\mathrm{pH} 6.5,7,7.5)$ and carbonate/bicarbonate $(\mathrm{pH} \mathrm{8.0,}$ 8.5) were used to train $(\mathrm{n}=3)$ the algorithm $(\mathrm{pH} \mathrm{5.0,6,6.5,7,}$ $7.5,8.0,8.5)$ and to predict $\mathrm{pH}$ with the trained algorithm $(\mathrm{pH}$ $5.5,6.25,6.75,7.25,7.75,8.25)$. To create soil extracts with varying $\mathrm{pH}, 5 \mathrm{~g}$ of soil was mixed with $25 \mathrm{ml}$ of buffer. The $\mathrm{pH}$ was then adjusted to the same $\mathrm{pH}$ as the buffers described above for $\mathrm{pH}$ predictions. Samples were thoroughly mixed for 1 minute and left to settle for 30 minutes.

\section{Nanoparticle suspensions and filter paper preparation}

Colloid GNP solution was concentrated 40 times by centrifuging (13000 RCF; $\left.30 \mathrm{~min} ; 20{ }^{\circ} \mathrm{C}\right)$. Algorithm training was performed using a $2 / 3$ times serial dilution and ranged from $37.5 \mathrm{nM}$ to $2.195 \mathrm{nM}$. For predictions a $2 / 3$ times serial dilution from $31.25 \mathrm{nM}$ to $2.74 \mathrm{nM}$ was used. For CB a $10 \mathrm{mg} / \mathrm{mL}$ dispersion was made in $\mathrm{H}_{2} \mathrm{O}$ and sonicated 30 minutes, then 
diluted to a $2 \mathrm{mg} / \mathrm{mL}$ dispersion and sonicated again for 10 minutes. For the training set a two times serial dilution was obtained from $2 \mathrm{mg} / \mathrm{ml}$ to $0,03125 \mathrm{mg} / \mathrm{mL}$ dispersion. For predictions a two times serial dilution was made from a 1.5 $\mathrm{mg} / \mathrm{ml} \mathrm{CB}$ dispersion up to $0.0117 \mathrm{mg} / \mathrm{mL}$.

\section{Sample preparation and picture capturing}

From GNP and CB dilutions $5 \mu \mathrm{L}$ was dropped on filter paper $(n=3)$, dried and photographed. $\mathrm{pH}$ strips $(\mathrm{n}=3)$ were immersed in buffer/soil extract, dried and photographed after 40 seconds. LFA test strips $(n=2)$ were photographed after an exposure of 10 minutes to $100 \mu \mathrm{L}$ of extract. Pictures were taken from 5,7 and $13 \mathrm{~cm}$ distance without digital zoom and from $5 \mathrm{~cm}$ with full digital zoom. The flashlight was always on as this reduces illumination variation ${ }^{31}$. Various layers $(0-4)$ of optical diffuser were taped in front of the LED light source to investigate if light diffusion could improve predictions and background correction. Performance of the prediction algorithm under various background illuminations was also tested. Here training was done under room light conditions and predictions under room light conditions, dark background conditions (in a closed windowless laboratory), indirect sunlight conditions (in a windowsill on a cloudy day) and direct sunlight conditions (in a windowsill under full sunlight). The performance of the algorithm under these conditions were compared with the performance when the algorithm was trained in a black cardboard box $(11 \times 11 \times 5 \mathrm{~cm})$ made for the Huawei (Figure S1) and used to predict in that same cardboard box. All images were acquired with the developed app under a white background (A4 paper sheet). Background images were taken before capturing a sample image to perform background correction on each image to reduce illumination variation error.

\section{Commercial lateral flow assay}

Cow milk was spiked into pure home-produced goat milk at $5,2.5,1.25,0.625,0.313,0.156,0.078,0.04$ and $0.02 \%$ and used as a training set. For predictions, goat milk was spiked with $3.75,1.88,0.47,0.23$ and $0.12 \%$. The test strips were immersed in 2 drops of sample diluted with 3 drops of provided dilution solution.

\section{Algorithm development}

Image background correction was performed following ${ }^{36}$. Briefly, a two-pixel wise system was used which allows pixel wise correction of each point in an image by a previously taken background image. RGB values of the input image were transferred into CieLab and HSV values using OpenCV library ${ }^{37}$. A previously developed algorithm was used as the prediction algorithm for colour/intensity quantification with slight modifications ${ }^{38}$. The algorithm is based on polynomial regression:

$$
\mathrm{h}_{\theta}(\mathrm{x})=\sum_{\mathrm{i}=0}^{\mathrm{N}} \sum_{\mathrm{j}=0}^{\mathrm{N}-\mathrm{i}} \sum_{\mathrm{k}=0}^{\mathrm{N}-\mathrm{i}-\mathrm{j}} \theta_{\mathrm{ijk}} \mathrm{x}_{1}^{\mathrm{i}} \mathrm{x} \hat{\mathrm{i}}_{2}^{\mathrm{k}}
$$

Where $\theta$ is the polynomial parameter vector, $\mathrm{N}$ the order number and $\mathrm{X} 1, \mathrm{X} 2$ and $\mathrm{X} 3$ the input colour values of the channels used for any combination of 3 channels with $\mathrm{X}_{1-3}$ being the respective single colour channels of the colour spaces.

The model was modified into the following forms to be compatible with two and single channel colour spaces:

$$
\begin{aligned}
& \mathrm{h}_{\theta}(\mathrm{x})=\sum_{\mathrm{i}=0}^{\mathrm{N}} \sum_{\mathrm{j}=0}^{\mathrm{N}-\mathrm{i}} \theta_{\mathrm{ij}} \mathrm{x}_{1}^{\mathrm{i}} \mathrm{x}_{2}^{\mathrm{i}} \\
& \mathrm{h}_{\theta}(\mathrm{x})=\sum_{\mathrm{i}=0}^{\mathrm{N}} \theta_{\mathrm{i}} \mathrm{x}_{1}^{\mathrm{i}}
\end{aligned}
$$

$\mathrm{N}$ was changed from the third up to the sixth order for each colour channel combination by running prediction simulations $(n=3)$ on captured images in Visual Studio Code. The order resulting in the lowest MAE on the prediction set was chosen for each channel combination and kept constant thereafter.

\section{Smartphone application workflow}

A custom developed smartphone application (Figure 1) was used to capture all images and works as follows. Captured images are saved in JPEG (750*1000 pixels) and send to a server where the background correction is performed and the regression function optimized for the set channel combination with the training set (Figure 1a). A background image is linked to the sample images to allow pixel wise background correction and needs to be taken before sample images (Figure 1b). The prediction algorithm can be trained for the prediction of various assays through labelling by an authorized user (Figure 1c). Images were labelled by drawing a box in the relevant region (within which pixel values are averaged) and typing in a numerical analyte concentration value (Figure 1d). For predictions, the user draws a box in the relevant area of the image (Figure1e). The average colour channel values of the background corrected pixels in the box are then used by the trained prediction algorithm to predict the concentration of the analyte.
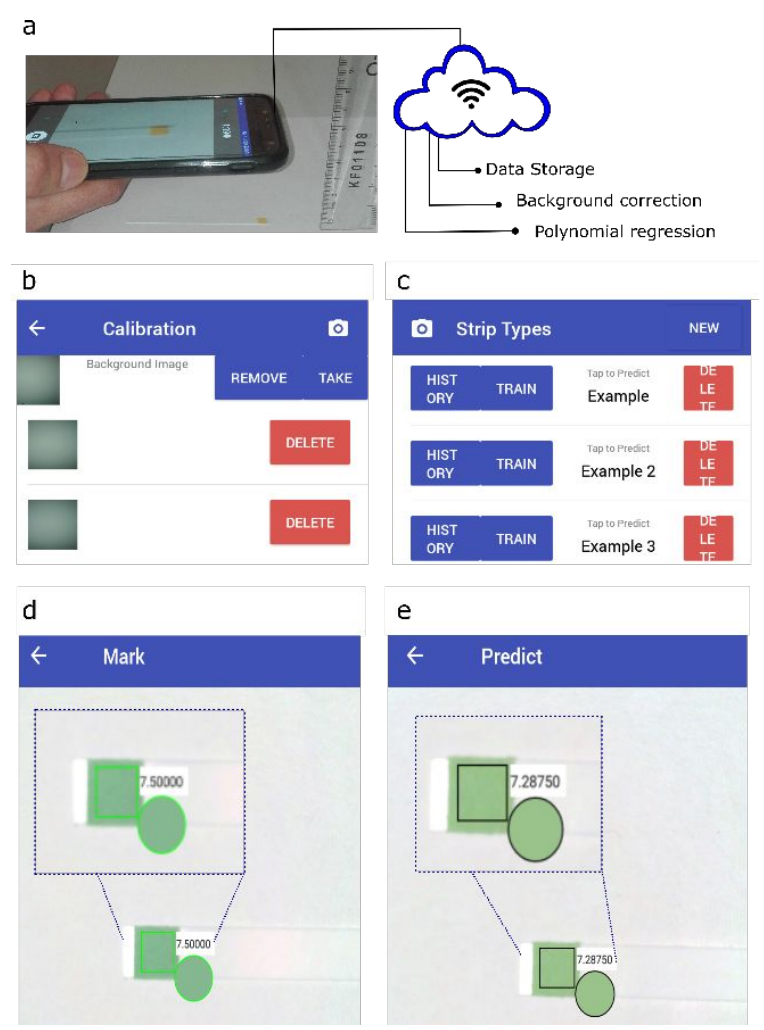

Figure 1. Development of the application. (a) Overview of the process. Pictures are taken without additional equipment. The image is autofocused in the app. Data is sent to a server where images are stored and where background correction and algorithm training/interpolation is performed. (b) Background images are taken in the calibration tab and are automatically linked to sample images for illumination correction. (c) Various assays can be created, trained and used to predict values in the app allowing the user to utilize the app for various systems. (d-e) Screengrabs of $\mathrm{pH}$ strip images used for training (d) and prediction (e). Boxes are 
drawn manually and used to enter a training value (d) or obtain a prediction (e).

\section{RESULTS AND DISCUSSION}

\section{Distance and optical diffuser optimization}

Pictures were taken of filter paper with varying concentrations of GNP at fixed distances and zoom $(13,7$, or 5 $\mathrm{cm}$ without digital zoom or at $5 \mathrm{~cm}$ with digital zoom) using LAC to optimize the distance between the camera and the assay. This experiment showed that prediction errors were similar for all distances and all concentrations chosen except for full digital zoom were larger errors were observed at lower concentrations (Figure 2a). Since different phone models allow autofocus at varying distances and to facilitate handheld imaging it was decided to take images at a distance between 5 and $13 \mathrm{~cm}$ from here-on depending on the phone model used. Furthermore, underestimation of GNP concentrations above $25 \mathrm{nM}$ was observed. This is likely due to saturation at concentrations above $25 \mathrm{nM}$. Thus, concentrations above $25 \mathrm{nM}$ GNP were not used further. Usefulness of optical diffuser to improve background correction and/or prediction algorithm performance was investigated. Predictions of zero points (white
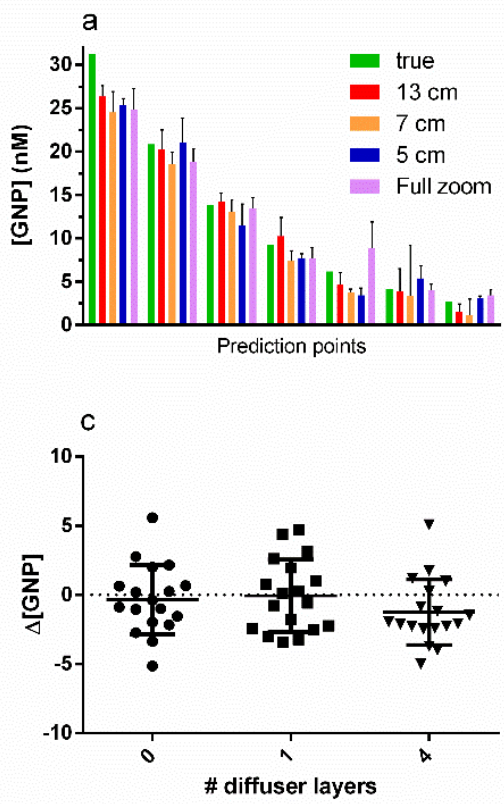

paper) were obtained with various layers of diffuser plastic taped to the LED light to test background correction performance. One-way ANOVA showed no significant difference in zero predictions. However standard deviations (STD) were clearly different $(\mathrm{p}<0.0001)$ with low STDs when no diffuser was used (Figure $2 \mathrm{~b}$ ). Estimations of the prediction set were obtained at 6 varying concentrations $(n=3)$ to compare the performance of the prediction algorithm using different layers of diffuser. Predictions per diffuser layer were pooled for all concentrations tested by deducting the true values from the predicted values ([ $\Delta \mathrm{GNP}]$; Figure $2 \mathrm{c})$. A one-way ANOVA on these results showed no significant difference in the prediction performance. STDs did also not differ significantly. Thus, no optical diffuser was used from here-on. Next performance of the prediction algorithm using a Huawei and Tablet in LAC colour space was tested for [GNP] prediction. The scatter plot obtained (Figure 2d) showed optimal $\mathrm{R}^{2}$ values for the linear regression with the Huawei (0.9625) and acceptable for the Tablet (0.9090). Thus, the algorithms showed promise in these tests for colour intensity measurements.
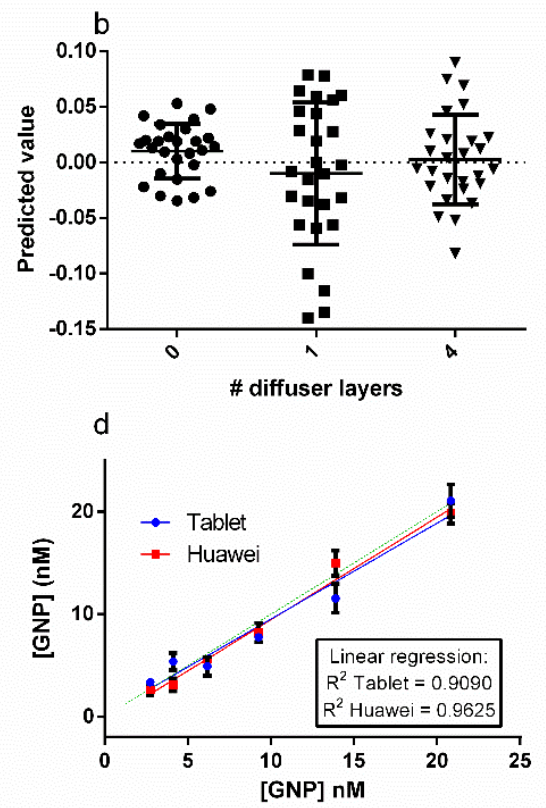

Figure 2. Distance and optical diffuser optimization. (a) Predictions ( $\mathrm{n}=3$ ) of [GNP] at 7 concentrations (range $31.25-2.75 \mathrm{nM}$ ) obtained with images taken at various distances and zoom (see legend). Green bars indicate true GNP concentrations on the tested papers. (b) Scatter plot of the zero values on white paper at various positions $(n=27)$ with 0,1 and 4 layers of optical diffuser used. (c) Scatter plot of [GNP] predictions $(\mathrm{n}=18$; range of $25-2.75 \mathrm{nM}$ ) with 0,1 and 4 layers of optical diffuser used. All predictions were scaled around zero by deducting the true values from each prediction. (d) Scatter plot of predicted [GNP] concentrations ( $\mathrm{n}=3$ per concentration) versus true concentrations using no optical diffuser or strict distance (between $5-13 \mathrm{~cm}$ ) using a Huawei and a Tablet. $\mathrm{R}^{2}$ values of the linear regression functions is indicated. Green dashed line indicates the slope of a perfect linear correlation. All predictions were done using LAC colour space.

\section{Channel combination performance in one smartphone.}

Visual studio code was used to calculate MAEs on concentration predictions for [GNP], [CB] $(n=18)$ and $\mathrm{pH}$ $(\mathrm{n}=15)$ using all possible single channels of RGB, HSV and LAC colour space and all non-repeating combinations of two and three of those channels (Figure 3a, c and e). The obtained MAEs of the 129 tested combinations clearly show that error is largest in predictions done with single channels (red points). Which of the single channels has the largest error is colour system specific. For instance, for [GNP] prediction R produces highest error, for $[\mathrm{CB}$ ] it is $\mathrm{C}$ and for $\mathrm{pH} \mathrm{S}$. Moreover, for $\mathrm{pH} \mathrm{R}$ produces the smallest error for all single channels while $\mathrm{B}$ and $\mathrm{G}$ are the best single channels for $[\mathrm{CB}]$ and $[\mathrm{GNP}]$ prediction respectively. MAEs of predictions undertaken with combinations of 2 (blue points) and 3 (magenta/green points and orange stars) channels vary considerably between channel combinations. However, several combinations of 2 and 3 channels allow for lower MAEs on the predictions as achievable by any single channel for all three colour systems tested. Interestingly, one such combination of two channels that 
outcompetes the performance of all single channel tested for $\mathrm{pH}$ prediction is $\mathrm{AC}$, the combination that was mentioned as favourable elsewhere ${ }^{17}$. RGB and LAC combinations outperformed the best performing single channels for [GNP] and $\mathrm{pH}$ prediction while HSV showed slightly higher MAEs, outperforming the best single channel only slightly for [GNP] predictions. Moreover, RGB and LAC showed small difference in obtained MAEs in all tested systems and were at least in the better performing half of all combinations tested in all cases. However, several other channel combinations clearly outperformed all single channels and RGB, LAC and HSV for each colour system. Next variations in algorithm performance, which can occur by overfitting to local optima in the polynomial calibration curves, was checked by running the same predictions five times for the ten combinations with the lowest MAEs and RGB, HSV and LAC (Figure $3 \mathrm{~b}, \mathrm{~d}$ and f). Of the 30 novel channel combinations chosen only 3 were 2 channels combinations while 27 where 3 channel combinations, spanning 2 and even 3 colour spaces. Of the ten novel combinations for [GNP] prediction six (RGS, RGA, GSL, RGV, RSL, GSV) outperformed the classics (RGB, LAC and HSV) with MAEs between 20.7 \pm 1.1 (RGS) and 23.9 $\pm 4.3 \%$ (GSV) versus MAEs between $27.8 \pm 3.6$ (LAC) and $32.6 \pm 6.4 \%$ (HSV) for the classics. Equally, the LOD of the best performing novel combination (RGS) was considerably improved (1.76 nM) compared with the LOD of the best performing classic (LAC; $2.34 \mathrm{nM})$. For $[\mathrm{CB}]$ predictions nine out of the ten novel combinations outperformed the classics (MAEs between 19.7 \pm $2.8 \%$ (BSC) and $33.9 \pm 11.0 \%$ (RHS) versus $36.3 \pm 13.2 \%$ (HSV) and $55.3 \pm 18.7 \%(\mathrm{RGB})$ ). For $\mathrm{pH}$ predictions six out of ten novel combinations outperformed the classics (MAEs between $0.82 \pm 0.02 \%$ (RVL) and $1.31 \pm 0.86 \%$ (SLA) versus $1.44 \pm 0.22 \%$ (LAC) and $2.20 \pm 1.21 \%(\mathrm{HSV}))$. Another observation is the considerably lower MAEs on $\mathrm{pH}$ prediction compared to MAEs on [GNP] and [CB] prediction. A reason for this may be that the replica variation is most likely considerably larger for the handmade paper strips when compared with the replica variation of the commercialized $\mathrm{pH}$ strips. Another reason for this may be a more limited effect of background illumination variation on the measurement of colour change instead of intensity change.
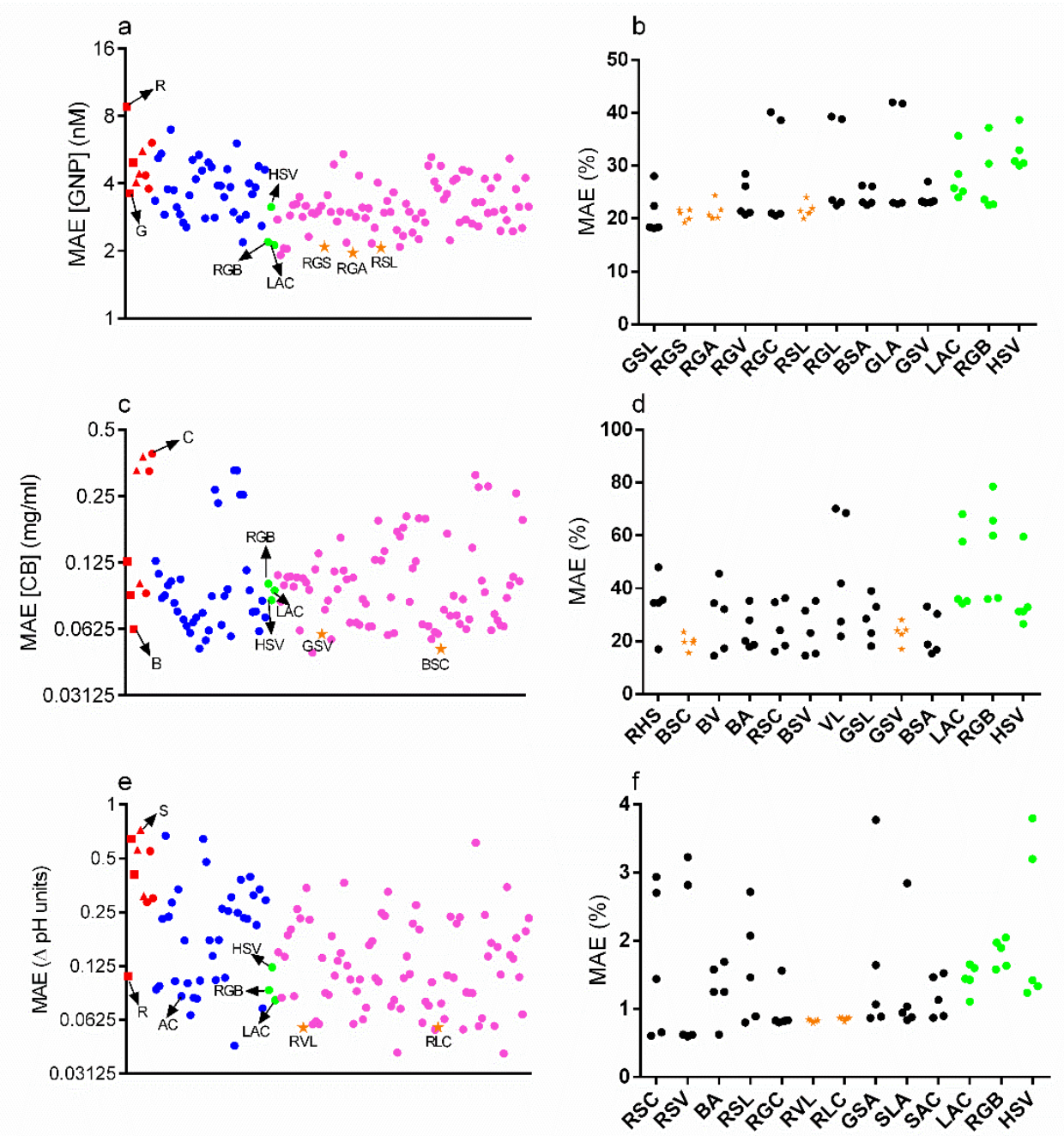

Figure 3. Prediction performance of random channel combinations. (a) Average MAEs (nM) on [GNP] predictions ( $\mathrm{n}=18$ per point) for single channels and all possible non-repeating channel combinations. (b) Average MAEs (\%) on [GNP] prediction ( $\mathrm{n}=18$ per point, 5 algorithm runs per channel combination) of RGB, LAC, HSV and the 10 best novel combinations identified in (a). (c) Average MAEs $(\mathrm{mg} / \mathrm{ml})$ on $[\mathrm{CB}]$ predictions $(\mathrm{n}=18$ per point) for single channels and all possible non-repeating channel combinations. (d) Average MAEs (\%) on [CB] prediction ( $\mathrm{n}=18$ per point, 5 algorithm runs per channel combination) of RGB, LAC, HSV and the 10 best novel combinations 
identified in (c). (e) Average MAEs ( $\Delta \mathrm{pH}$ units) on $\mathrm{pH}$ predictions ( $\mathrm{n}=15$ per point) for single channels and all possible non-repeating channel combinations. (f) Average MAEs (\%) on $\mathrm{pH}$ prediction ( $\mathrm{n}=15$ per point, 5 algorithm runs per channel combination) of RGB, LAC, HSV and the 10 best novel combinations identified in (e). In (a), (c) and (e) red balls are LAC, red squares RGB and red triangles HSV single channels. Best and worst performing single channels for each target are indicated. Blue balls are all non-repeating random combinations of 2 channels. Magenta balls are all non-repeating random combinations of 3 channels except HSV RGB and LAC which are green in all figure panels. Orange stars indicate novel combinations chosen for further experiments.

\section{Channel combination performance in various phones}

The best novel (orange stars Figure 3) and classic (green balls Figure 3) combinations were selected for colour/intensity quantification with four phones (Huawei, iPhone, Tablet, and Samsung) to test if the performance of novel/classical combinations is phone and illumination source dependent (Figure 4). Two-way ANOVAs on these MAEs (Table S1) show that both smartphone model and channel combination cause significant differences in MAEs for [GNP], [CB] and $\mathrm{pH}$ predictions $(p<0.0001)$ while interactions were significant $(p<0.0001)$ for all but [CB] predictions. Thus, for [GNP] and $\mathrm{pH}$ predictions it depends on the channel combination chosen if phone model has an effect on the MAE. Post-hoc analyses show many significant differences in MAE between phone models within channel combinations for [GNP] prediction. However, these differences are most prominent when HSV and LAC are used (Table S2). Post-hoc analyses on the pH MAEs between phone models within channel combinations showed that there were no significant differences for the channels combinations LAC, RVL, RLC and RGB. With LAC having the highest $p$ - values $(p>0.5$; Table S3). Thus, the phone model used influences the prediction efficiency of a given channel combination in many but not all cases. Moreover, choice of phone model does not affect MAEs stronger for novel channel combinations as classical ones in any of the systems tested. Indeed, all novel channel combinations showed less MAE variation between phones as HSV for [GNP] prediction (posthoc main row effect $p<0.0001$ ) and RVL as well as RLC showed less MAE variation between phones as HSV and RGB for $\mathrm{pH}$ prediction (post-hoc main row effect $p<0.0001$ ). This being said, the inter-phone variation reduction (and thus robustness enhancement) for $\mathrm{pH}$ prediction was less prominent as it did not differ from the inter-phone variation using LAC. Overall, variance in MAEs between phone models was limited for two ([GNP] and $\mathrm{pH}$ prediction) of the three reported colour systems for the best functioning channel combinations. Measurement error caused by source illumination variation and other differences between phone models showed inferior to error caused by channel choice and was minimized by optimizing the colour channel combination used.
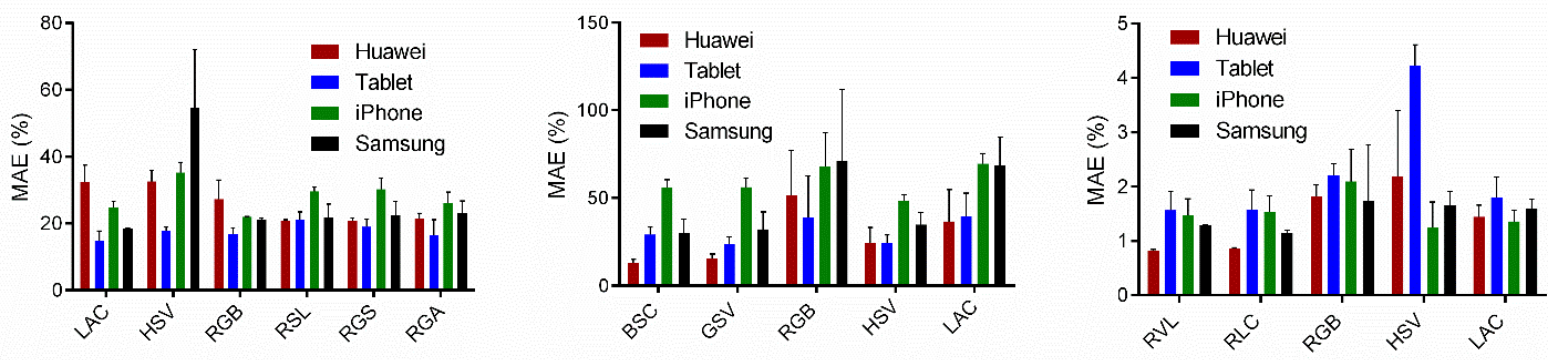

Figure 4. Prediction performance of classic and novel channel combinations with various phones. Left; MAEs for [GNP] prediction ( $\mathrm{n}=18$ ). Middle; MAEs for [CB] prediction ( $\mathrm{n}=18)$. Right; MAEs for $\mathrm{pH}$ prediction $(\mathrm{n}=15)$.

\section{Background illumination and light-shielding box}

GNPs were chosen as the better assay for intensity quantification since less inter-phone variance in MAEs was observed for [GNP] as for [CB] prediction. Moreover, MAEs on $[\mathrm{CB}]$ prediction were quite high (in some cases $>100 \%$ ) which might indicate issues with replicate variance due to the manual dropping process. Thus, $[\mathrm{CB}]$ prediction was not used for further experiments. MAEs of predictions with the classic and previously selected novel channel combinations for [GNP] and $\mathrm{pH}$ prediction under various illumination conditions was determined (Figure 5). The two-way ANOVAs (Table S4) on these MAEs were both highly significant $(p<0.0001)$ for phone model, channel combination and interaction. For $\mathrm{pH}$ predictions (Figure 5; top) the smallest effect of illumination variation on the MAEs was observed when RVL or RLC was used with the only significant differences in the post-hoc simple effects within colour spaces analyses for RVL being box versus direct sunlight and for RLC box and room light versus direct sunlight $(p<0.05$; Table S5). MAEs for predictions with classic channel combinations for $\mathrm{pH}$ showed higher variations caused by illumination variation with HSV showing the most prominent variation (Figure 5; Table S5). Moreover, STDs appear lower for RVL and RLC compared to the classics for MAEs on $\mathrm{pH}$ prediction. Equally, STDs are mostly lower on MAEs obtained with RSL and RGS compared to the classics for [GNP] prediction. However, this effect is less prominent for RGA. Interestingly, the smallest effect of illumination variation on the MAEs for [GNP] prediction was observed when RGB was used (albeit with slightly higher STDs) instead of the novel combinations. This can indicate that the novel combinations used were highly sensitive for assay specific variations (colour change or intensity change). Thus, changes effecting the illumination caused little difference in MAEs for the assay optimized for colour change $(\mathrm{pH})$ but large difference in MAEs for intensity change ([GNP] prediction) when background illumination was varied and no light-shielding box was used. These observations support the assumption made that unintended background illumination variation may have a more substantial effect on intensity measurement as on colour 
change, thus causing higher MAEs for [GNP] compared to $\mathrm{pH}$ prediction. For $\mathrm{pH}$ predictions the light-shielding box was found of little use for the better functioning channels (LAC, RVL and RLC) with only significant effect found being box versus direct sunlight $(\mathrm{p}<0.05)$. Interestingly, the MAEs obtained for [GNP] predictions using a box were higher than MAEs obtained in room light for LAC and RSL $(p<0.01)$ and in the dark for LAC $(\mathrm{p}<0.01)$ (Table S6). This can be caused by unequal light distribution in the box effecting the background illumination correction. However, when background illumination increases (indirect sunlight) the box allows for significantly lower MAEs when RGS or RGL are used $(\mathrm{p}<$ 0.01 ) while no significant difference is found with other channel combinations (likely due to higher STDs on the MAEs for these other channels). For direct sunlight versus box there is a significant difference when HSV, RSL, RGS or RGA is used $(\mathrm{p}<0.0001$; Table S6).
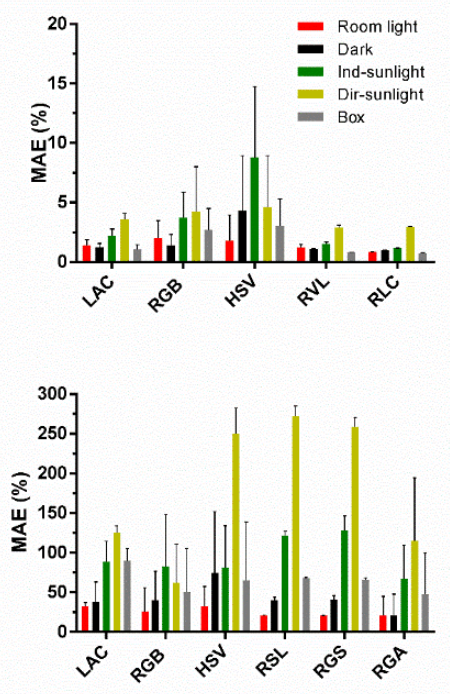

Figure 5. The effects of a light-shielding box and illumination variations on [GNP] and $\mathrm{pH}$ prediction using classic and novel channel combinations. Top; MAEs on $\mathrm{pH}$ prediction $(\mathrm{n}=15)$. Bottom; MAEs on [GNP] prediction

\section{Quantification of a commercial lateral flow assay and $\mathrm{pH}$ prediction of soil samples}

A lateral flow assay that uses red latex beads for colorimetric detection of cow milk protein was used to quantify cow milk in goat milk with the classic and novel channel combinations identified for red colour (GNP) quantification while the $\mathrm{pH}$ of buffered soil samples was predicted using classic and novel channel combinations identified for colour change (Figure 6). R2 values and $95 \%$ slope confidence intervals for the linear regressions using these channel combinations (Figure 6a, c) as well as LODs are shown in Table S7. For cow milk quantification good R2 values, 95\% slope confidence intervals and LOD were obtained for RGA (confidence interval 0.71 to 1.06; R2=0.96; LOD $0.16 \%$ cow milk). All other channel combinations had large slope confidence intervals with HSV performing the poorest in terms of slope interval (0.19-1.68) with a LOD of $0.25 \%$ cow milk. LAC performed poorest in terms of R2 (0.756) and LOD ( $0.51 \%$ cow milk) but had a slightly better slope confidence interval $(0.32$ to 1.17$)$. Next BSA channel combination (which equally showed low MAEs with little variation for [GNP] quantification in Figure 3) was added to the analyses. BSA (confidence interval 0.81 to 1.17 ; $\mathrm{R} 2=0.97$; LOD $0.15 \%$ cow milk) outperformed the classic channel combinations (Table S7) and RGA. Moreover, MAEs on BSA and RGA (Figure 6b) were significantly lower in posthoc analyses $(p<0.05)$ on the one-way ANOVA $(p<0.0001)$ compared to MAEs obtained with the classic (except for the RGA-HSV comparison) while no significant difference was observed between RGA and BSA MAEs (Table S8). Linear regressions for $\mathrm{pH}$ prediction in soil extracts (Figure $6 \mathrm{c}$ ) showed excellent slope confidence intervals, R2 values and LODs for the novel channel combinations (RVL 1.01 to 1.10 confidence interval, R2 $=0.997, \mathrm{LOD} \mathrm{pH} 5.15$; RLC 1.04 to 1.12 confidence interval, R2 $=0.997$, LOD $\mathrm{pH} 5.12$ ) shortly followed by LAC (confidence interval 0.99 to1.21, R2 $=0.980$, LOD 5.17). HSV and RGB showed poorer performance with HSV preforming poorest (Table S7). The one-way ANOVA on MAEs obtained for $\mathrm{pH}$ prediction was highly significant $(\mathrm{p}<0.0001)$. Post-hoc analyses showed that RVL and RLC obtained MAEs that did not significantly differ. However, both novel channel combinations outperformed all classic combinations $(\mathrm{p}<0.001$; Table S9). Moreover, all channel combinations clearly outperformed HSV in terms of MAEs on the predictions $(\mathrm{p}<0.0001)$. Interestingly, the absolute difference in MAEs between the channel combinations is smaller for $\mathrm{pH}$ prediction as for cow milk quantification but the relative difference in error reduction is similar. The MAE with the best classic colour space for cow milk quantification was HSV (MAE is 58\%). The MAE of the best functioning novel channel combination (BSA) was $36 \%$. Thus, a $38 \%$ reduction in MAE was observed by using this combination instead of HSV. For $\mathrm{pH}$ prediction in soil the best functioning classic colour space was RGB (MAE 2.08\%). For the best functioning specific channel combination (RLC) the MAE was $1.31 \%$. Thus a $37 \%$ reduction in MAE was observed by using this combination instead of RGB.

\section{CONCLUSION}

Combining multiple channels for the prediction of colour and intensity change based assays reduced mean average prediction errors. Perhaps most interestingly it was discovered that effective assay quantification is not dependent of how a channel combination allows a correct description of true colours but rather if a channel combination allows description of assay specific changes. Thus, smartphone-based colorimetrics can be optimized by combining channels from various colour spaces for a specific assay. Moreover, some of these combinations allow reduction of inter-phone variance when compared with the classic channel combinations HSV, RGB and LAC (CieLAB) and clearly outperformed the classics and single channels in terms of prediction error for all tested colour systems. Additionally, it was shown that the optimum channel combinations were similar for assays with similar red intensity colour changes ([GNP] quantification and LFA quantification). Thus, other assays with red intensity-based colour changes such as plasmonic ELISAs may equally perform well with these channel combinations. However, optimum combinations for assays based on colour change are different from combinations for colour intensity change. Moreover, various intensity change assays have different optimum channel combinations (although CieLab and RGB outperformed HSV in both systems). Thus, it is recommended to optimize the channel combinations used for the development of specific assays with a different colour base 
as the colour bases discussed here. No improvements in performance were found in the case of optical diffusers. Equally, use of a light-shielding box can be avoided if measurements are not taken in direct sunlight and pixel wise background illumination correction is used for assays based on colour change. For intensity prediction, the lowest MAEs are found when images are taken under room light or in the dark without using a box. However, to allow measurements in direct or indirect sunlight a box is required to reduce error. Finally, these findings were used to optimise the prediction algorithm of the smartphone application for the quantification of a lateral flow assay for milk adulteration and for $\mathrm{pH}$ prediction in soil with $\mathrm{pH}$ strips. Once the colour channel combination was optimized the algorithmic computation was fixed for these assays. Thus, $\mathrm{pH}$ or LFA assay quantification by the end-user can simply be performed by selecting the assay in the app. This will load the correct algorithm automatically. The assay is then quantified by taking a background and assay image and drawing a box around the test area in the assay image. Thus, the procedure can be performed by users without analytical expertise.
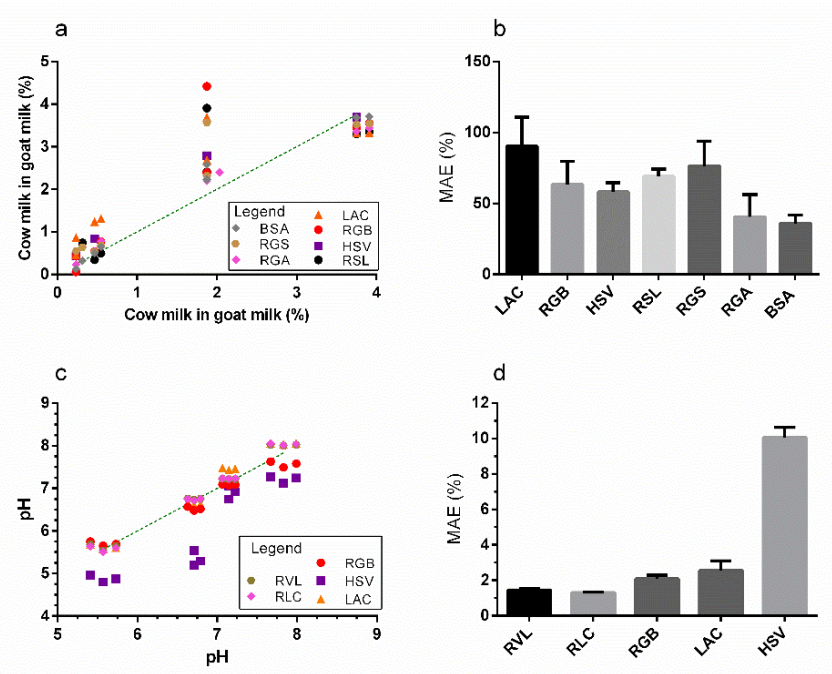

Figure 6. Cow milk quantification in goat milk and $\mathrm{pH}$ prediction of soil samples. (a) Scatter plot for the quantification of cow milk spiked into goat milk $(\mathrm{n}=2)$ using previously identified novel channel combinations for [GNP] prediction and classic channel combinations. (b) MAE $\%(n=8)$ on the predictions shown in (a). (c) Scatter plot for the prediction of $\mathrm{pH}$ values in soil ( $\mathrm{n}=3$ ) using previously identified novel channel combinations for $\mathrm{pH}$ prediction in buffer and classic channel combinations. (d) MAE \% ( $\mathrm{n}=8)$ on the predictions shown in (c)

\section{ASSOCIATED CONTENT}

\section{Supporting Information}

The Supporting Information is available free of charge on the ACS Publications website. Additional figure, materials, statistical analyses, tables S1-9 with results of statistical tests.

\section{AUTHOR INFORMATION}

\section{Corresponding Author}

Joost Laurus Dinant Nelis. Email: J.Nelis@qub.ac.uk

\section{Author Contributions}

The manuscript was written through contributions of all authors. All authors gave approval to the final version of the manuscript.

\section{ACKNOWLEDGMENT}

This project has received funding from the European Union's Horizon 2020 research and innovation program under the Marie Sklodowska-Curie grant agreement No 720325. We thank Ilyana Nelis for providing home produced pure goat milk.

\section{REFERENCES}

(1) Sicard, C.; Glen, C.; Aubie, B.; Wallace, D.; Jahanshahi-Anbuhi, S.; Pennings, K.; Daigger, G. T.; Pelton, R.; Brennan, J. D.; Filipe, C. D. M. Tools for Water Quality Monitoring and Mapping Using Paper-Based Sensors and Cell Phones. Water Res. 2015, 70, 360-369.

(2) Wei, Q.; Nagi, R.; Sadeghi, K.; Feng, S.; Yan, E.; Ki, S. J.; Caire, R.; Tseng, D.; Ozcan, A. Detection and Spatial Mapping of Mercury
Contamination in Water Samples Using a Smart-Phone. ACS Nano 2014, 8 (2), 1121-1129.

(3) Levin, S.; Krishnan, S.; Rajkumar, S.; Halery, N.; Balkunde, P. Monitoring of Fluoride in Water Samples Using a Smartphone. Sci. Total Environ. 2016, 551-552, 101-107.

(4) Ross, G. M. S.; Bremer, M. G. E. G.; Nielen, M. W. F. ConsumerFriendly Food Allergen Detection: Moving towards Smartphone-Based Immunoassays. Anal. Bioanal. Chem. 2018, 410 (22), 5353-5371.

(5) Nelis, J.; Elliott, C.; Campbell, K. "The Smartphone's Guide to the Galaxy": In Situ Analysis in Space. Biosensors 2018, 8 (4).

(6) Rateni, G.; Dario, P.; Cavallo, F. Smartphone-Based Food Diagnostic Technologies: A Review. Sensors 2017, 17 (6).

(7) Xu, X.; Akay, A.; Wei, H.; Wang, S.; Pingguan-Murphy, B.; Erlandsson, B.; Li, X.; Lee, W.; Hu, J.; Wang, L.; Xu, F. Advances in Smartphone-Based Point-of-Care Diagnostics. Proc. IEEE 2015, 103 (2), 236-247.

(8) Ozdalga, E.; Ozdalga, A.; Ahuja, N. The Smartphone in Medicine: A Review of Current and Potential Use Among Physicians and Students. $J$ Med Internet Res 2012, 14 (5), e128.

(9) Smith, T.; Guild, J. The C.I.E. Colorimetric Standards and Their Use. Trans. Opt. Soc. 1931, 33 (3), 73-134.

(10) Melorose, J.; Perroy, R.; Careas, S. Color Spaces for Computer Graphics 1978. Statew. Agric. L. Use Baseline 2015 2015, 1, 20-25.

(11) Billmeyer, Fred W., and Saltzman, M. Principles of Color Technology.; J. Wiley \& Sons, Ed.; Wiley-Interscience: New York, 1981. (12) García, A.; Erenas, M. M.; Marinetto, E. D.; Abad, C. A.; de OrbePaya, I.; Palma, A. J.; Capitán-Vallvey, L. F. Mobile Phone Platform as Portable Chemical Analyzer. Sensors Actuators B Chem. 2011, 156 (1), 350-359.

(13) Lopez-Ruiz, N.; Curto, V. F.; Erenas, M. M.; Benito-Lopez, F.; Diamond, D.; Palma, A. J.; Capitan-Vallvey, L. F. Smartphone-Based Simultaneous $\mathrm{PH}$ and Nitrite Colorimetric Determination for Paper 
Microfluidic Devices. Anal. Chem. 2014, 86 (19), 9554-9562.

(14) Oncescu, V.; O'Dell, D.; Erickson, D. Smartphone Based Health Accessory for Colorimetric Detection of Biomarkers in Sweat and Saliva. Lab Chip 2013, 13 (16), 3232-3238.

(15) Lee, S.; Oncescu, V.; Mancuso, M.; Mehta, S.; Erickson, D. A Smartphone Platform for the Quantification of Vitamin D Levels. Lab Chip 2014, 14 (8), 1437-1442.

(16) Oncescu, V.; Mancuso, M.; Erickson, D. Cholesterol Testing on a Smartphone. Lab Chip 2014, 14 (4), 759-763.

(17) Shen, L.; Hagen, J. A.; Papautsky, I. Point-of-Care Colorimetric Detection with a Smartphone. Lab Chip 2012, 12 (21), 4240-4243.

(18) Ravindranath, R.; Periasamy, A. P.; Roy, P.; Chen, Y.-W.; Chang, H.T. Smart App-Based on-Field Colorimetric Quantification of Mercury via Analyte-Induced Enhancement of the Photocatalytic Activity of TiO2--Au Nanospheres. Anal. Bioanal. Chem. 2018, 410 (18), 4555-4564.

(19) Tania, M. H.; Lwin, K. T.; Shabut, A. M.; Najlah, M.; Chin, J.; Hossain, M. A. Intelligent Image-Based Colourimetric Tests Using Machine Learning Framework for Lateral Flow Assays. Expert Syst. Appl. 2020, 139, 112843.

(20) Motalebizadeh, A.; Bagheri, H.; Asiaei, S.; Fekrat, N.; Afkhami, A. New Portable Smartphone-Based PDMS Microfluidic Kit for the Simultaneous Colorimetric Detection of Arsenic and Mercury. RSC Adv. 2018, 8 (48), 27091-27100.

(21) Li, F.; Hu, Y.; Li, Z.; Liu, J.; Guo, L.; He, J. Three-Dimensional Microfluidic Paper-Based Device for Multiplexed Colorimetric Detection of Six Metal Ions Combined with Use of a Smartphone. Anal. Bioanal. Chem. 2019, 411 (24), 6497-6508.

(22) Kim, H.; Awofeso, O.; Choi, S.; Jung, Y.; Bae, E. Colorimetric Analysis of Saliva-Alcohol Test Strips by Smartphone-Based Instruments Using Machine-Learning Algorithms. Appl. Opt. 2017, 56 (1), 84.

(23) Su, K.; Qiu, X.; Fang, J.; Zou, Q.; Wang, P. An Improved Efficient Biochemical Detection Method to Marine Toxins with a Smartphone-Based Portable System-Bionic e-Eye. Sensors Actuators, B Chem. 2017, 238, $1165-1172$.

(24) Chen, Y.; Fu, G.; Zilberman, Y.; Ruan, W.; Ameri, S. K.; Zhang, Y. S.; Miller, E.; Sonkusale, S. R. Low Cost Smart Phone Diagnostics for Food Using Paper-Based Colorimetric Sensor Arrays. Food Control 2017, 82, 227-232.

(25) Tsagkaris, A. S.; Pulkrabova, J.; Hajslova, J.; Filippini, D. A Hybrid Lab-on-a-Chip Injector System for Autonomous Carbofuran Screening.

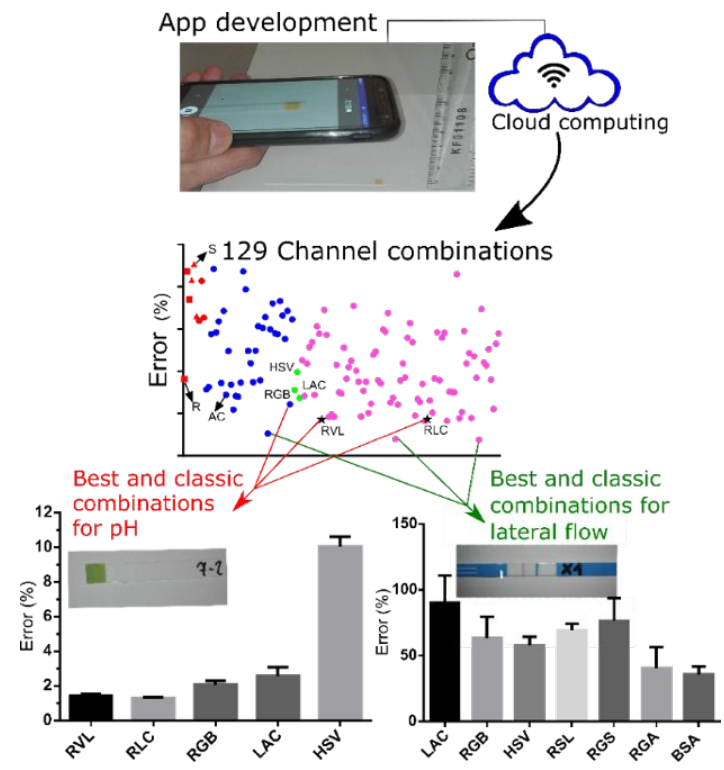

For Table of Contents Only
Sensors 2019, 19 (24).

(26) Nelis, J. L. D.; Bura, L.; Zhao, Y.; Burkin, K. M.; Rafferty, K.; Elliott, C. T.; Campbell, K. The Efficiency of Color Space Channels to Quantify Color and Color Intensity Change in Liquids, PH Strips, and Lateral Flow Assays with Smartphones. Sensors 2019, 19 (23).

(27) Hosu, O.; Lettieri, M.; Papara, N.; Ravalli, A.; Sandulescu, R.; Cristea, C.; Marrazza, G. Colorimetric Multienzymatic Smart Sensors for Hydrogen Peroxide, Glucose and Catechol Screening Analysis. Talanta 2019, 204, 525-532.

(28) Fang, J.; Qiu, X.; Wan, Z.; Zou, Q.; Su, K.; Hu, N.; Wang, P. A Sensing Smartphone and Its Portable Accessory for On-Site Rapid Biochemical Detection of Marine Toxins. Anal. Methods 2016, 8 (38), 6895-6902.

(29) Roda, A.; Michelini, E.; Zangheri, M.; Di Fusco, M.; Calabria, D.; Simoni, P. Smartphone-Based Biosensors: A Critical Review and Perspectives. TrAC - Trends Anal. Chem. 2016, 79, 317-325.

(30) Urusov, A. E.; Zherdev, A. V; Dzantiev, B. B. Towards Lateral Flow Quantitative Assays: Detection Approaches. Biosensors 2019, 9 (3).

(31) Kong, T.; You, J. B.; Zhang, B.; Nguyen, B.; Tarlan, F.; Jarvi, K.; Sinton, D. Accessory-Free Quantitative Smartphone Imaging of Colorimetric Paper-Based Assays. Lab Chip 2019, 19 (11), 1991-1999.

(32) Kim, S. C.; Jalal, U. M.; Im, S. B.; Ko, S.; Shim, J. S. A SmartphoneBased Optical Platform for Colorimetric Analysis of Microfluidic Device. Sensors Actuators, B Chem. 2017, 239, 52-59.

(33) Turkevich, J. Colloidal Gold. Part I. Gold Bull. 1985, 18 (4), 125-131. (34) Haiss, W.; Thanh, N. T. K.; Aveyard, J.; Fernig, D. G. Determination of Size and Concentration of Gold Nanoparticles from UV. Anal. Chem. 2007, 79 (October), 4215-4221.

(35) Mcvey, C.; Logan, N.; Thanh, N. T. K.; Elliott, C.; Cao, C. Unusual Switchable Peroxidase-Mimicking Nanozyme for the Deter- Mination of Proteolytic Biomarker. Nano Res. 2019, 12 (1), 1-8.

(36) Zhao, Y.; Elliott, C.; Zhou, H.; Rafferty, K. Pixel-Wise Illumination Correction Algorithms for Relative Color Constancy under the Spectral Domain. 2018 IEEE Int. Symp. Signal Process. Inf. Technol. ISSPIT 2018 2019, 638-643.

(37) Bradski, G. Kaehler, A. Learning OpenCV: Computer Vision with the OpenCV Library; O’Reilly Media, Inc., 2008.

(38) Zhao, Y.; Choi, S. Y.; Nelis, J. L. D.; Zhou, H.; Cao, C.; Campbell, K.; Elliott, C.; Rafferty, K. Smartphone Modulated Colorimetric Reader with Color Subtraction. 2019 IEEE Sensors. Accept. Publ. 1-4. 